Annais of Otology Rhono logy \& Loxyngology 114(7):533-538.

2005 Annals Publishing Company, All rights reserwd.

\title{
Audiologic Features of Norrie Disease
}

\author{
Chris Halpin, PhD; Grace Owen, ScD; Gustavo A. Gutiérrez-Espeleta, PhD; \\ Katherine Sims, MD; Heidi L. Rehm, PhD
}

\begin{abstract}
Objectives: Norrie disease is an X-linked recessive disorder in which patients are born blind and develop sensory hearing loss in adolescence. The hearing loss associated with Nomie disease has been shown in a genetically altered knockout mouse to involve dysfunction of the stria vascularis; most other structures are preserved until the later stages of the disease. The objective of this study was to characterize the audiologic phenotype of Norrie disease for comparison with the pathophysiologic mechanism.

Methods: The design combined two series of clinical audiologic evaluations, with special attention to speech intelligibility.

Results: The audiologic results for 12 affected individuals and 10 carriers show that patients with Norrie disease retain high speech inteligibility scores even when the threshold loss is severe.

Conclustons: The cochlear mechanism - failure of the stria vascularis - accounts for some of the higher values in the wide distribution of spech scores in cases with similar pure tone audiograms.
\end{abstract}

Key Words: audiometry, NDP gene, Norrie disease, speech intelligibility, stria vascularis.

\section{INTRODUCTION}

Norrie disease is an $\mathrm{X}$-linked recessive disorder caused by point mutations in the $N D P$ gene at Xp11.4.1,2 Affected individuals are born blind, with pseudogliomas of the eyes, and exhibit various degrees of mental and emotional dysfunction. The audiologic features of Norrie disease were described previously by Parving and Warburg. ${ }^{3}$ These authors found normal hearing at birth, with the onset of hearing loss in eady adolescence and progression to anacusis late in life. They also found good word recognition scores ( $80 \%$ correct or better for monosyllables). Further study by this same group indicated a cochlear site of lesion. ${ }^{4}$ Molecular genetic studies have identified the Norrie disease gene (NDP),, 2 characterized the protein (norrin), ${ }^{5}$ and generated a knockout mouse. ${ }^{6}$ Rehm et al ${ }^{7}$ used light and confocal microscopy to identify the specific sites of cochlear disease in the mouse model of Norrie disease. These authors showed gross developmental abnormalities of the stria vascularis in the knockout mice by 3 months of age compared with normal littermates. Most other structures in the cochlea appeared normal in affected mice at this age. Longitudinal analysis showed that the initial effects in the cochlea occur in the stria; neurons and hair cells are affected in the later stages of life. This article presents the audiologic results of two series of patients with Norrie disease.

\section{METHODS}

Two clinical investigations are presented. The first reflects a series of patients in the United States. After informed consent procedures were completed, 6 affected individuals (male), 2 carriers (mothers), and 4 unaffected family members ( 2 fathers and 2 female siblings) were tested. Diagnosis of Norrie disease had previously been made in these farnilies and was confirmed by molecular analysis showing point mutations in the $N D P$ gene. Most patients were evaluated by standard audiometry for pure tones. ${ }^{8}$ Speech intelligibility was evaluated with 50 -item CID W22 word lists on compact disc. ${ }^{9}$ One affected adult, institutionalized for severe mental disability, was evaluated by threshold auditory brain stem response audiometry. One other affected adult, also severely mentally disabled, was evaluated behaviorally by a conditioning procedure. (For an overview of audiologic methods in genetic investigations, see the 1995 article by Halpin. ${ }^{10}$ )

The second investigation took place in Costa Rica. After giving informed consent, subjects were evalu-

From the Department of Otology and Laryngology, Har vard Medical School (Halpin), the Department of Audiology, Massachusetts Eye and Ear Infirmary (Owen), the Department of Neurology, Massachusetts General Haspital, Har vard Medical Schol (Sims), and the Department of Pathology, Harvard Medical School (Rehm), Boston, Massachusetts, and Escuela de Biologia, Instituto de Investigaciones en Salud. Universidad de Costa Rica, San Jose, Casta Rica (Gutierrez-Espeleta). The Costa Rican investigation was funded by the National Institutes of Health National Institute on Deafness and Other Communication Disorders (Fant RO1 DCO2281).

Correspondence: Chris Halpin, PhD, Dept of Audiology, Masachusetts Eye and Ear Infirmary, 243 Charles St, Boston, MA 02114. 


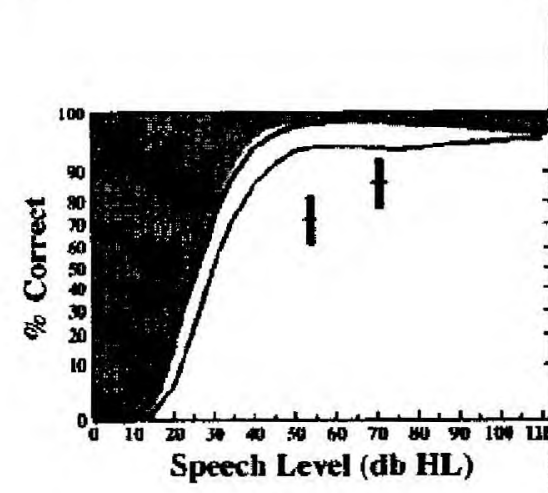

A

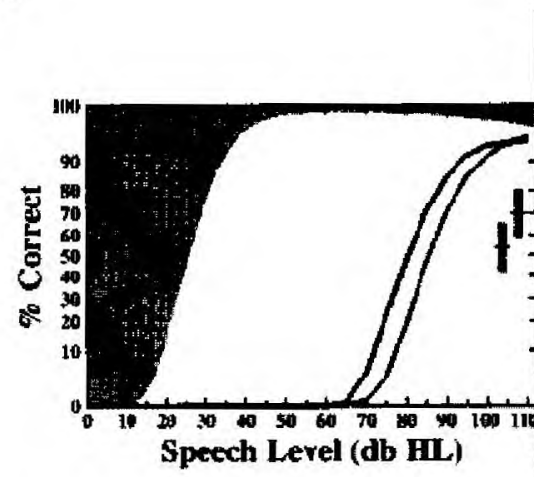

B

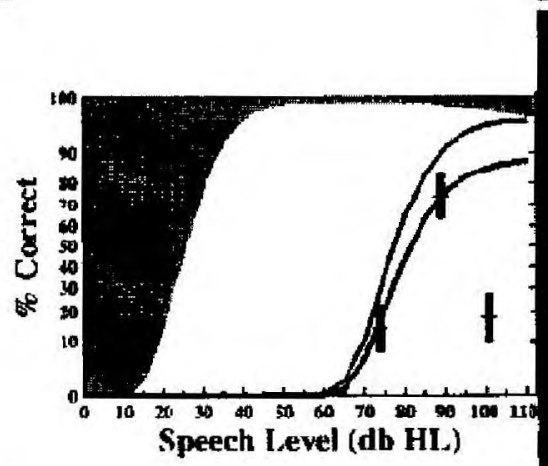

C

ated by a certified audiologist who was a native speaker of Spanish (G.O.). Medical and molecular diagnosis of Norrie disease had also been previously confirmed in this large family. ${ }^{11}$ Six affected male individuals, 8 female carriers, and 20 unaffected family members were tested. The subjects were evaluated by standard pure tone audiometry in a quiet, but not sound-treated, room. Objective intensity calibration was guaranteed by daily use of a sound level calibrator (BA-201-25, Quest Technologies, Oconomowoc, Wisconsin) that never showed variations within standard one-third-octave filter bands greater than 1
Fig 1. Output of Haryard Audiometer Operating System zoftware. ${ }^{15}$ Audiology case screen consists of standard pure tone audiogram (on right) and speech intelligibility space (on left). This space consists of speech signal intensity on horizontal axis from 0 to $110 \mathrm{~dB}$ hearing level, and percent correct on yertical axis. Clinical test results (CID W22) are plotted by level and score in this space such that horizontal tick on data point bars (red, right; blue. left) represent score, and extent of bars represents 95\% critical difference from another score. 14 Vertical axis is arcsine transformed such that critical difference bars will remain same size even though variance of speech scores changes, given range in which score is found. Ogive (" $\mathrm{s}$ ") curves represent performance function predicted by Articulation Index given right and left ear thresholds. ${ }^{13}$ Similar ogive is calculated for condition with all thresholds at limit of normal hearing (20 dB hearing level) and forms outer boundary of normal range (shaded gray for reference). This Figure shows example cases from both $\mathbf{A}, \mathbf{B}$ ) United States and C) Costa Rica.
dB sound pressure level. In situ biocalibration of the test room for the effect of noise resulted in audiograms of normal listeners with no thresholds higher than $20 \mathrm{~dB}$ hearing level. This level of test sensitivity is sufficient to reliably separate Norrie disease (or any other) effects from normal hearing subjects. An affected 2-year-old boy was evaluated by a conditioning procedure approprate for his age group.

In CostaRica, speech intelligibility was evaluated in adults with 50-item Spanish bisyllable lists delivered by compact disc. The lists used were selected 
Fig 2. Output of Harvard Audiometer Operating System software. 15 Unumual cases. A) Patient has near-normal apeech ability with severe hearing loss, B) Least-affected individual C) Female obligate carrier (mother of patients in Figs $1 B$ and $2 A$ ) is only carrier with hearing loss of 10 carriers evaluated.

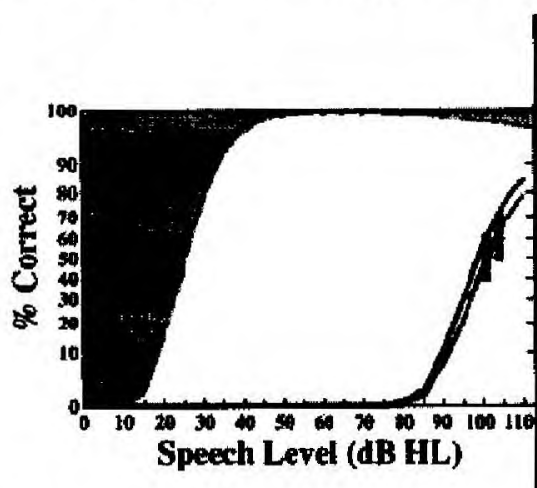

A
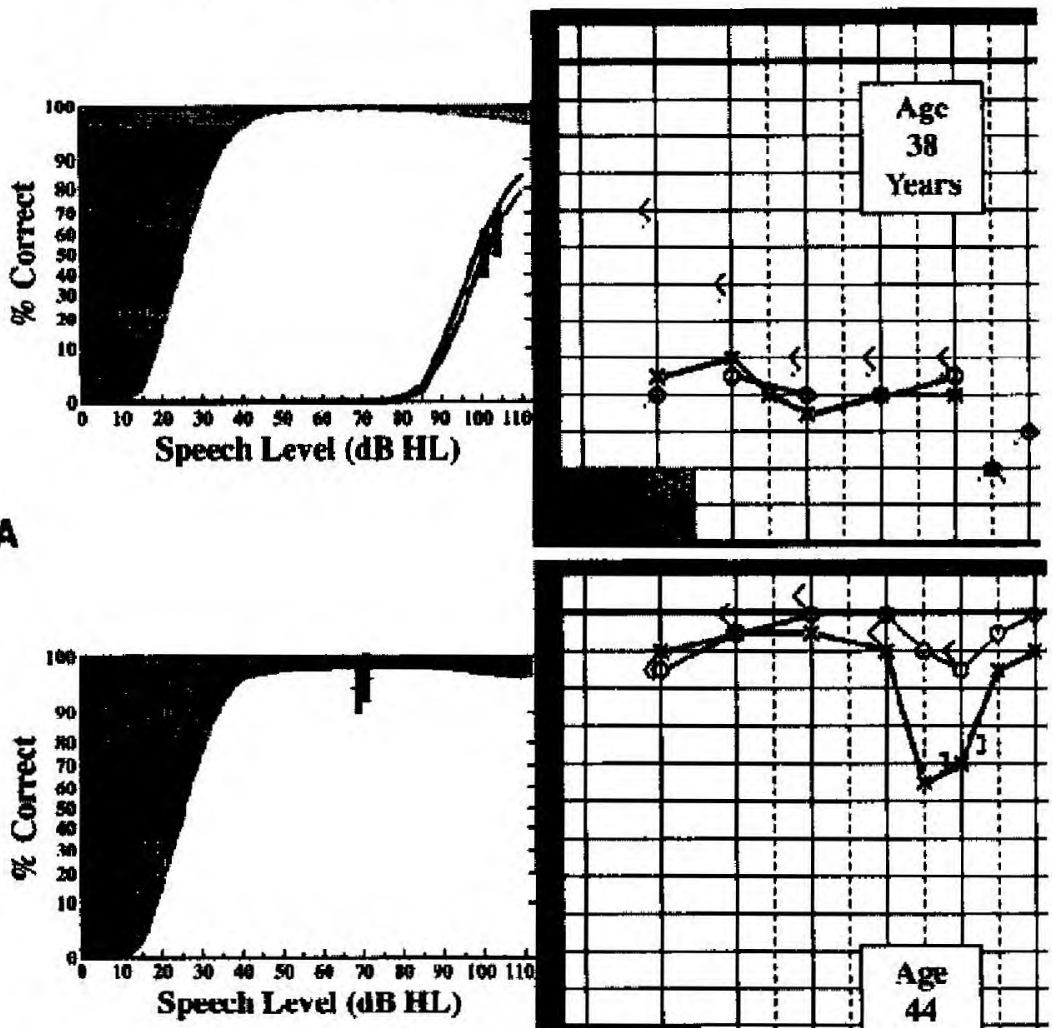

B
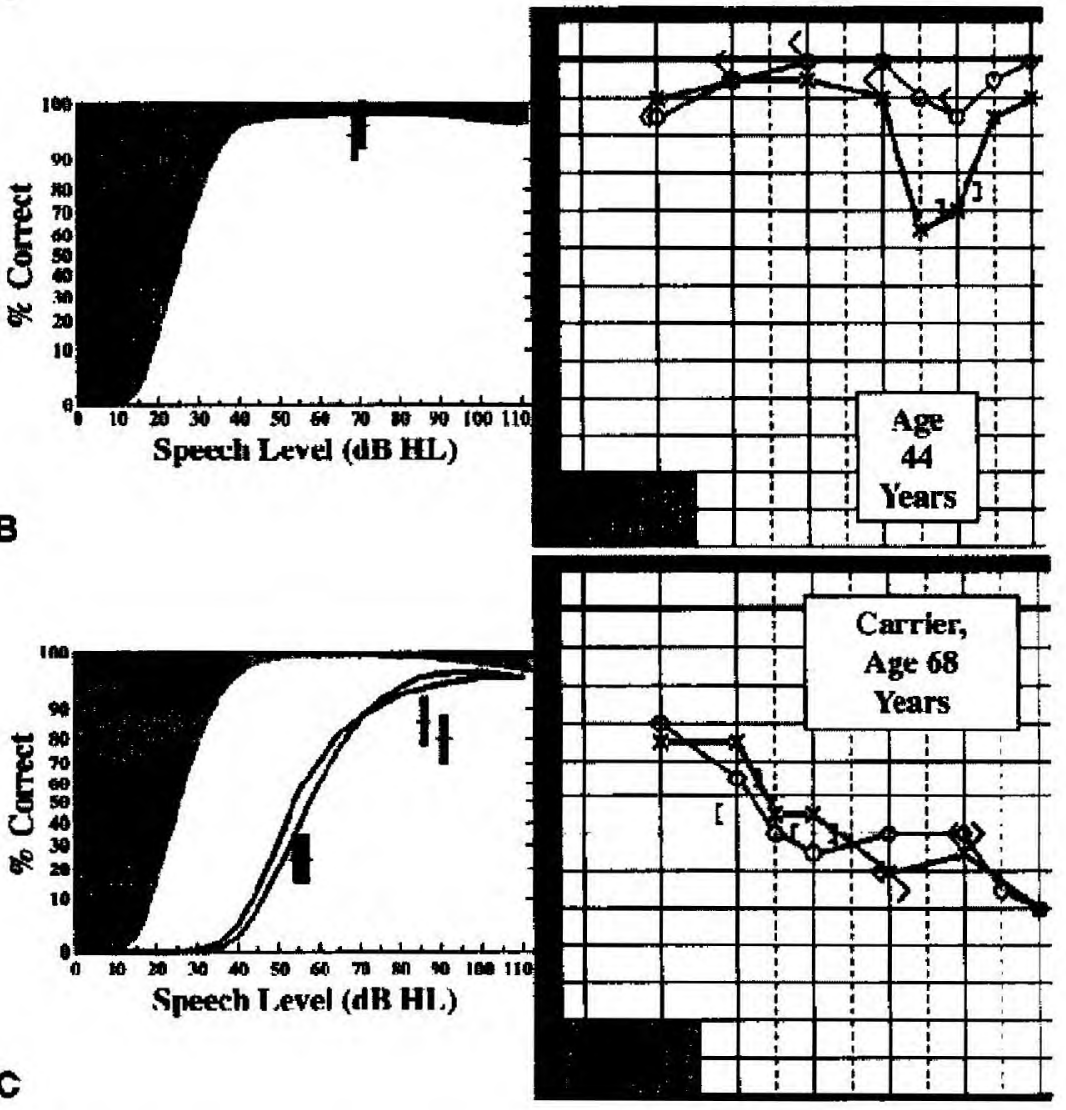

because their published performance/intensity function ${ }^{12}$ had a practically identical slope and intercept to that of the CID W22 lists used in the United States. The expected values and variability of these lists ane equivalent, and they are plotted interchangeably within the speech intelligibility space on the audiograms in Figs $1^{13-15}$ and 2. 15

\section{RESULTS}

Figure 1 shows 3 cases that illustrate the general progression of the hearing loss. Figure 1A reflects a 14-year-old individual with an intragenic deletion in exon 2. (Mutation identifications were performed in the laboratory of author $\mathrm{K}$. Sims.) His documented hearing was normal up to 7 years of age. This audiogram shows the high-frequency emphasis and asymmetry often reported in the earlier stages. Speech intelligibility was also affected, but his scores did not fall below $78 \%$ correct. The sensory hearing loss in these two series (US and Costa Rica) progressed to a relatively stable state during the third and fourth decades of life, with features illustrated by Fig 1B. In this 40-year-old individual (mutation R109X), the threshold loss had become severe (approximately 70 $\mathrm{dB}$ hearing level), flat, and symmetric, with preservation of word recognition near $80 \%$ correct. Figure 


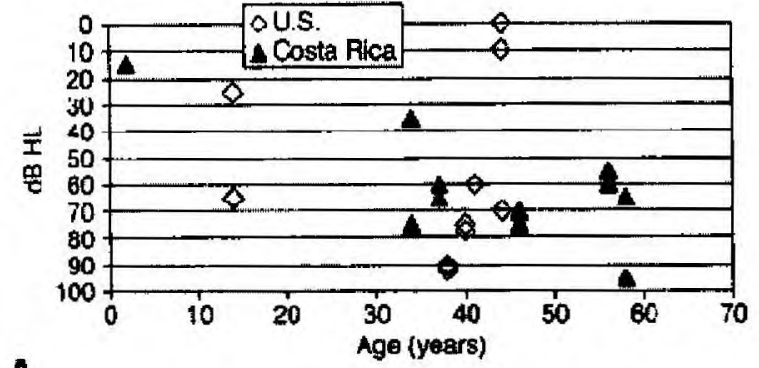

A

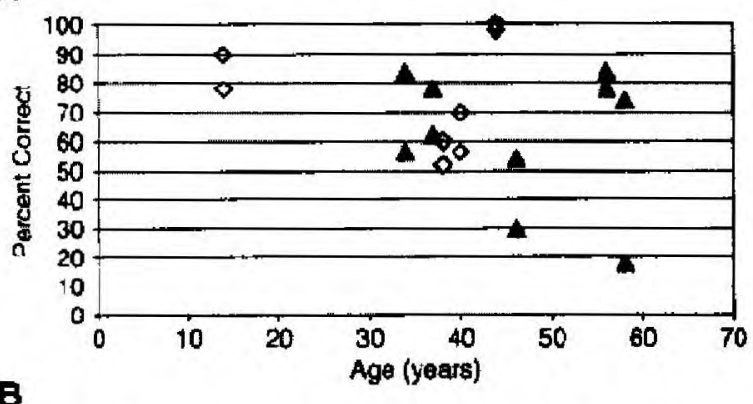

$\mathbf{B}$

Fig 3. Summary of clinical te st results. A) Thre sholds at $2 \mathrm{kHz}$ versus age for each ear of each affected individual. B) Word recognition scores (CID W22, and equivalent Spanish lists) for each ear by age. Exceptions to this are individuals evaluated by auditory brain stem responses and conditioning audiometry, who are given single data point for threshold and none for speech intelligibility.

1C shows the clinical results of the oldest individual (58 years of age, L61F). ${ }^{11}$ In this case, the symmetry between ears seen in middle-aged individuals is breaking down. Although the right ear still exhibits the severe flat hearing loss with speech discrimination at $74 \%$, the left ear shows a further threshold loss and an extreme decrease in speoch intelligibility (18\%).

Figure 2 shows 3 cases that reflect some findings of individual interest. The results in Fig 2A (38 years of age, the brother of patient in Fig 1B; R109X) should be noted for unusual interpretation in light of speech score analysis using the Articulation Index. ${ }^{13} \mathrm{Al}$ though the absolute scores ( $60 \%$ right and $52 \%$ left) could be considered poor, these scores are not significantly different from the predicted values for the Articulation Index calculation. ${ }^{14}$ (In Fig $2 \mathrm{~A}$, the variance bars touch the ogive curves. ${ }^{15}$ ) This means that a normal listener would get a similar score with the same limited amount of audible speech. Therefore, this case, with its low score, does not detract from the general trend for very good speech intelligibility in the presence of severe cochlear disease.

Figure $2 \mathrm{~B}$ shows the individual with the least hearing loss. At this age ( 44 years), he would be expected to exhibit a severe flat loss on both sides (as in Fig 1B). Although his sensory loss resembles a " $4-\mathrm{kHz}$ notch," this patient had no history of any exposure to gunfire or industrial noise. Interestingly, this pa- tient has a mutation in the $N D P$ gene $(\mathrm{R} 121 \mathrm{Q})$, which has been observed to be milder in terms of both the eye phenotype ${ }^{16}$ and the lack of hearing loss reported in one family. ${ }^{17}$ Further studies will be needed to investigate specific mutations that may be indicative of normal or near-normal hearing.

Finally, Fig $2 \mathrm{C}$ depicts the data of the mother of 2 affected individuals (Figs $1 \mathrm{~B}$ and $2 \mathrm{~A}$ ), who is therefore an obligate carrier. This was the only 1 of the 10 carriers in these two series who had a severe, symmetric sensorineural hearing loss similar to that seen in Norrie disease. This is a very unusual and possibly coincidental finding, as hearing loss in carriers has not been seen in previous reports. ${ }^{18,19}$ It is also possible that the hearing loss is a manifestation of nonrandom $X$-inactivation (or chance segregation of such cells) in the ear tissue. Such a case involving eye disease in a female $N D P$ gene mutation carrier has been described ${ }^{20}$

A summary of the combined clinical test results for all affected individuals can be found in Fig 3 . These results show complete penetrance of sensory hearing loss in all affected individuals 14 years of age and older. (One US individual, shown in Fig 2B, is affected at frequencies not graphed.) The graph includes threshold data from 2 mentally disabled individuals. One of these data points (at 41 years of age) was interpolated from a $65 \mathrm{~dB}$ normal hearing level auditory brain stem response to a broadband click including $2 \mathrm{kHz}$ within its bandwidth, and another (at 44 years of age) contributes a single $2-\mathrm{kHz}$ threshold found in the sound field. The youngest individual ( 2 years of age) contributes a single $2-\mathbf{k H z}$ value in the normal range, found with a conditioning procedure. None of these 3 individuals contribute to the speech data (Fig 3B). Although the progressive loss shows a high-frequency emphasis and notable asymmetry in the earlier stages, the progression seems to result in a sy mmetric, bilateral, flat sensory loss for patients above 35 years of age. This loss seems generally stable for 2 to 3 decades by both these data and clinical history. The speech intelligibility scones (Fig 3B) are fairly high (mean, 78\%), given the severity of the hearing loss. These are also fairly stable up to 58 years of age.

\section{DISCUSSION}

The results of this investigation confirm and ex. tend those reported by Parving and Warburg. ${ }^{3}$ In terms of threshold, the hearing losses are sensorineural, mild, asymmetric, and high-frequency in adolescence, and progress to a severe, symmetric, flat loss around 35 years of age. The previous audiometric and medical reports include several individuals around 25 years 
of age and older than 50 years of age. ${ }^{3,21}$ This study provides a number of audiograms in the intervening age range, and these results indicate that the disease does not progress steadily, but generally achieves a steady state of severe loss from about 35 years of age to nearly 60 years of age. There is some evidence from this study, that of Parving and Warburg, ${ }^{3}$ and the reported temporal bone from a 77 -year-old individual $^{22}$ that suggests that a very severe degeneration follows at about 60 years of age. Whereas Warburg 23 initially estimated the penetrance of sensorineural hearing loss at $33 \%$, several other reports showed loss in all tested individuals above the age of puberty. $3,21,24$ In this study as well, all men over 13 years of age showed clear sensorineural loss, indicating $100 \%$ penetrance of this feature.

Norrie disease serves as a rare example in which audiologic speech intelligibility can be studied within a group whose cochlear pathophysiology arises from the same mechanism, and that mechanism is indicated by a supporting mouse model. Speech intelligibility can be a more informative challenge to a dysfunctional cochlea than the binary detection task of the pure tone audiogram, and therefore can be more informative about the underlying state of the ongan of Corti. ${ }^{25}$ Although the audiograms of cochleas with different pathophysiologic mechanisms may be quite similar, ${ }^{26}$ the differences in clinical speech scores are more likely to be informative regarding different underlying disorders (and prognoses for hearing aids). ${ }^{27}$ This is especially true if at least one such mechanism allows for remarkably high scores, as is the case in Norrie disease. Clinicians routinely observe similar audiograms with much lower scores, and possibly the different mechanisms in such cases may be further quantified as similar models become available.

Some authors have suggested that all sensory hearing loss is the result of outer hair cell loss plus inner hair cell loss. ${ }^{28}$ Although not specifically stated, such an approach would suggest that spech intelligibility would be predictable from threshold audiogram values alone and that the range actually seen in the clinic could be attributed to poor test reliability. The results of this study clearly do not support this assertion, as they indicate the stria vascularis as at least one additional mechanism. With the clear possibility of complex interactions among the mechanisms resulting in both high and low scores, speech scores remain valuable in parsing individual cochlear dysfunction and should be measured and applied within clinical cases, rather than assumed to be predictable from pure tone threshold values.

In a more general sense, it is important to remember that "sensory hearing loss" is a symptom of a variety of cochlear dysfunctions and is not expected to give cohenent results when different pathophysiologic mechanisms are grouped together. In this study, molecular genetics, pathophysiology, and speech audiometry provide insight into a specific cochlear mechanism that limits patient performance. Similar collaborations in the future may allow separate study of other specific cochlear disorders.

Acknowledgments: The authors thank Dr Agaete Parving for providing information regarding the speech testing from previous studies, and Dr Joseph Pillion for providing data on one of the affected individuals. David Corey, $\mathrm{PhD}$, and colleagues also provided vahuable advice and support.

\section{REFERENCES}

1. Chen $Z Y$, Hendriks RW, Jobling MA, et al. Isolation and characterization of a candidate gene for Nomie disease. Nat $G$ net 1992;1:204-8.

2. Berger W, Meindl A, yan de Pol TJ, et al. Isolation of a candidate gene for Nonie disease by positional cloning. Nat Genet 1992;1:199-203. [Erratum in Nat Genet 1992;2:84.]

3. Parving A, Warturg M.Audiological findings in Norrie's di sease. Audiology 1977:16:124-31.

4. Parving A, Elberling C. Warturg M. Electrophysiological study of Nonrie's disease. An X-linked recessive trait with hearing loss, Audiology 1978;17:293-8.

5. Perez-Vilar J, Hill RL. Norrie disease protein (norrin) forms disulfide-linked oligomers associated with the extracellular matrix. J Biol Chem 1997:272:33410-5.

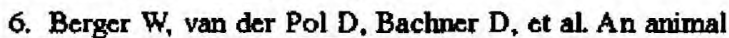
model for Norric disease (ND): gene targeting of the mouse ND gene. Hum Mol Genet 1996:5:5 1-9.

7. Rehm HL, Zhang DS, Brown MC, et al. Vascular defects and sensorineural deafness in a mouse model of Norrie discase. J Neurosci 2002;22:4286-92.
8. \$3.21. Methods for manual pure-tone threshold audiom etry. New York, NY: American National Standards Institute. 1978 R-1986.

9. Thornton A. Raffin M. Q/MASS. Vol 1. A recording of materials for speech auctiometry (compact disc). Minneapolis. Minn: Qualitone Inc, 1988.

10. Halpin C. Modem clinical methods in genetic hearing loss. Curr Opin Otolaryngol Head Neck Surg 1995;3:325-31.

11. Rehm HL, Gutierroz-Espeleta GA, Garcia R, et al. Norric disease gene mutation in a large Costa Rican kindred with a novel phenctype inchuting venous insufficiency. Hum Mutat 1997;9:402-8.

12. De Candenas M, Marrero V. Cuademo de logaaudiometria. Madrid, Spain: Universidad Nacional de Educacion a Distancia, 1994.

13. S3.5. Methods for calculation of the Articulation Index. New York, NY: American National Standards Institute, 1969.

14. Thornton AR. Raffin MJ. Speech-discrimination scores modeled as a binomial variable. I Speech Hear Res 1978;21:50718. 
15. Thomton A, Halpin C, Han Y, Hou Z. The Harvard Audometer Operating System [Software]. PaloAlto, Calif Applitech Inc, 1994.

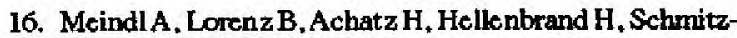
Valckenberg $\mathrm{P}$, Meitinger T. Missense mutations in the NDP gene in patients with a less severe course of Norric discase. Hum Mol Genet 1995;4:489-90.

17. Fuentes JJ, Wolpini V, Fernandez-Toral F, Coto E, Estivill $X$. Identification of two new missense mutations (K58N and R121Q) in the Norrie disease (ND) gene in two Spanish families. Hum Mol Genet 1993;2:1953-5.

18. Parving A, Schwartz M. Audiometric tests in gene carriers of Norrie's disease. Int I Pediatr Otorhinolaryngol 1991;21: 103-11.

19. Warburg M, Friedrich U, Blecker-Wagemakers L, Wienker TF, Gal A. Ropers HH. Norrie"s disease: delineation of carriers among daughters of obligate carriers by linkage analysis. Trans Ophthalmol Soc U K 1986;105:88-93.

20. Sims KB, Irvine AR, Good WV. Norrie disease in a family with a manifesting female carrier. Arch Ophthalmol 1997; 115:517-9.

21. Blodi FC. Hunter WS. Norrie's disease in North America.
Dac Ophthalmol 1969;26:434-50.

22. Nadol JB Jr, Eavey RD, Liberfarb RM, et al. Histopathology of the ears, eyes, and brain in Norrie's disease (oculoacousticocerobral degeneration). Am J Otolaryngol 1990;11: 112-24.

23. Warburg $M$, Norrie's disease. A congenital progressive oculo-acoustico-cerebral degeneration. Acta Ophthalmol (Copenh) Suppl 1966(suppl 89).

24. Hohmes LB. Nortie's disease: an X-linked syndrome of retinal malformation, mental retardation and deafness. J Pediatr 1971:79:89-92.

25. Hahin C. The tuming curve in clinical audiology. Am J Audiol 2002;11:56-64.

26. Schuknecht HF. Pathology of the ear. 2nd ed. Philadelphia. Pa: Lea and Febiger, 1993.

27. Halpin C. Thomton A, Hasso M. Low-frequency sensorineural loss: clinical evaluation and implications for hearing aid fitting. Ear Hear 1994;15:71-81.

28. Moore BC, Vickers DA. Plack CJ, Oxenham AJ. Interrelationship between different psychoacoustic measures assumed to be related to the cochlear active mechanism. J Acoust Soc Am 1999; 106:2761-78. 
Página 1 de 1

inisa

De: $\quad$ "Gustavo Gutiérrez Espeleta" <ggutier@biologia.ucr.ac.cr>

Para: "inisa" <inisa@cariari.ucr.ac.cr>

Enviado: Martes, 26 de Julio de 2005 06:13 p.m.

Adjuntar: 533.pdf

Asunto: NORRIE PDF

Para los efectos pertinentes, adjunto esta publicación.

Gustavo 